\title{
Polarization Diversity for SKA Wide-field Polarimetry
}

\author{
T.D. Carozzi ${ }^{1}$, G. Woan ${ }^{1}$, and R. Maaskant ${ }^{2}$ \\ 1 Dept. of Physics and Astronomy, University of Glasgow, Glasgow G12 8QQ, U.K. * \\ 2 Netherlands Institute for Radio Astronomy (ASTRON), P.O. Box 2, 7990 AA Dwingeloo, The Netherlands
}

Abstract. We look at how wide-field polarimetry with the SKA can be improved by using polarization diversity.

\section{Introduction}

One of the most promising features of the Square Kilometre Array (SKA) telescope will be its wide field of view (FoV). With a projected FoV of 250 square degrees (Schilizzi et al. 2007), the SKA will be a prime survey tool for radio astronomers, and considering the novelty of a wide-field telescope it is fitting that a conference be held on the wide-field science and technology for the SKA in which this paper is a contribution. In addition the SKA will be fully polarimetric, so provides unprecedented instantaneous wide field polarimetry. This will be achieved using dual-polarized, aperture array (AA) technology; a technology which has been employed by radio engineers for some time but which is relatively new to radio astronomy. Yet such wide field polarimetry is not just a novel technological capability, it will play a vital part in achieving many of the important scientific objectives of the SKA.

Indeed, out of the five core SKA key science projects (KSP), three of them, namely the "Epoch of reionisation" (KSP1), "Cosmic Magnetism" (KSP3), and "Strong field tests of gravity" (KSP4), are identified as being polarization purity drivers (Schilizzi et al. 2007, sect. 5.1.6). This means that, for these KSPs, a high degree of polarization purity is important. In addition, all of these KSPs will entail a survey over wide fields. It is therefore fair to say that all in all wide field polarimetry is very important to the SKA.

However, it is also fair to say that the AA technology employed by the SKA is yet to be validated for astronomical measurements, and one area that may prove difficult is precisely that of wide field polarimetry. The reason for this is simply that it is more difficult to maintain polarization purity over wide fields than narrow fields. Traditional radio astronomy methodology is to employ swivel mounted dishes with inherently narrow fields of view. For such telescopes planar cartesian geometry can safely be assumed (known as the telescope isoplanatic assumption) and any polarization impurities within the field of view are well known as the primary beam is nearly independent of the mechanical pointing. In contrast the AA technology is fix-mounted to the ground with no moving parts, and has an inherently wide field. For an AA telescope one must therefore

\footnotetext{
^ This work was supported by the European Commission Framework Program 6, Project SKADS, Square Kilometre Array Design Studies (SKADS), contract no 011938.
}

deal with a curved field and a primary beam which varies with (electronic) pointing.

The fact that the field is curved (ultimately a region of the celestial sphere) is basis for concern. The "hairy ball" theorem of topology tells us that it is fundamentally impossible to cover the sphere with a continuous single mapping of base vectors. Thus in antenna engineering, an isotropic antenna can never exist. All antennas must have at least one null, usually more, and one should not expect a uniform reception over large fields of view. For polarimetry based on dual-polarized elements, another relevant ramification of the theorem is that the far-field radiation vectors of the two polarized elements of a dual-polarized antenna cannot be orthogonal (and non-zero) in all directions, and so it may well be the case that the two radiation vectors are not linearly independent (i.e. they are degenerate) over the field of view making calibration impossible. The linear independence of the far-field radiation vectors of the dual-polarized elements is related to the polarization purity of the telescope, and thus the fundamental question here is can the SKA deliver sufficient polarization purity to fulfil its science goals?

The dual-polarized AA technology employed by the SKA performs polarimetry by extracting at most two tangential vectors on the sky that correspond to the two far-field radiation vectors of the dual-polarized element. However the polarimetric base vectors mapped onto the sky are not likely to be orthogonal (or non-null) over wide fields. ${ }^{a}$ The fundamental questions are therefore how well will the SKA perform wide field polarimetry based on AA technology, and will this be enough to satisfy its scientific goals?

These questions are not entirely new, after all, this is why the SKA specification lists cross-polarization ratios at the centre and at the edge of the field. What is recently starting to emerge though, is how much more difficult the challenge of calibrating the polarimetry over wide fields will be with the AA technology as compared with the more traditional dish approach. Hamaker raised concerns in his paper (Hamaker 2000) on the calibration of the SKA in light of what he called its "inherently strong instrumental polarization". In this paper, we will look at some of the problems facing wide field polarimetry, and we will mention the possibility of mitigating some of the risks involved in using the AA technology for wide field

\footnotetext{
a Note that no amount of beamforming can change this since the beamforming is a scalar operation. More on this in a later section.
} 

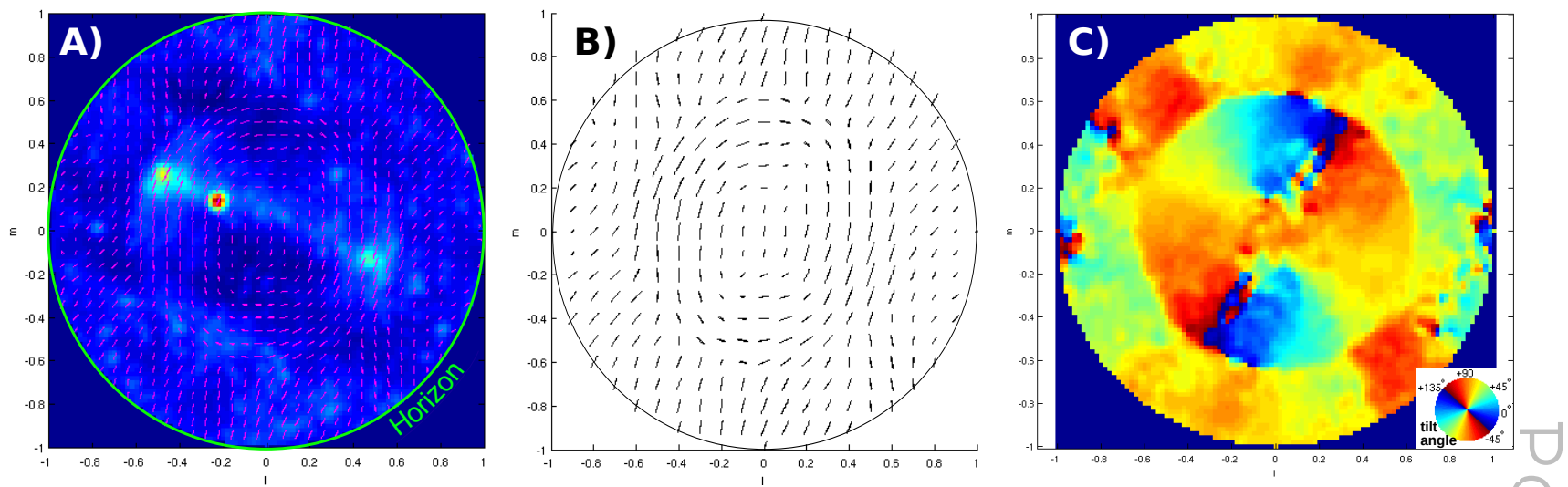

Fig. 1: Example of polarimetric aberrations in the LBA array of LOFAR CS10.

polarimetry using polarization diversity. Polarization diversity can be achieved in many ways, but here we will only consider varying the relative orientation of the AAs.

\section{Degeneracy in Wide-field Polarimetry}

Earlier we mentioned that, over wide enough fields, there must be some regions on the celestial sphere where the two far-field radiation vectors of a dual-polarized antenna ${ }^{b}$ are degenerate, and even if the polarimeter vectors are not completely degenerate, the may be almost degenerate. This is at least what we expect from theory, but is this really seen in practice? We now show examples of this in practice.

Before we can do that though, we must explain what we mean here by degeneracy. Degeneracy is a measure of the linear dependence between two vectors. As discussed in the paper Carozzi \& Woan (2009a), dual-polarized polarimeters are often polarimetrically aberrated over a wide field. That is, the state of polarization of an off-axis source will often be distorted. However, this aberration may not be significant if the polarimeter can be fully calibrated, but unfortunately, as shown in Carozzi \& Woan (2009b), aberrations generally cannot be removed entirely due to the presence of noise. The calibratability of the polarimeter in some direction was shown to be directly related to the polarimeter's intrinsic cross-polarization ratio (IXR), a quantity introduced in Carozzi \& Woan (2009b). The IXR is in turn directly related to the condition number of the Jones matrix of the polarimeter. Ultimately, the IXR sets the upper bound on the relative error in the total polarimetry of the polarimeter. Essentially the IXR is an invariant cross-polarization ratio, while the standard extrinsic crosspolarization ratio depends on the coordinate systems used for the polarimetry performed by the polarimeter.

\subsection{Examples of polarimetric aberrations over wide fields}

Fig. 1 shows an example of polarimetric aberrations in the Low-Band Antenna (LBA) (van Cappellen et al. 2007) array

\footnotetext{
b We will sometimes call these two vectors the polarimeter vectors.
}

of the LOFAR CS10. The data was taken on the 19 June 2007 by Stefan Wijnholds at $80.002 \mathrm{MHz}$. Fig. 1A) shows an all-sky raw image of the intensity plotted in direction cosine coordinates $(l, m)$. Overlayed on that image, and in Fig. 1B), is the polarization ellipses (in magenta) of the radiation on the sky at a subset of the grid points. Note that due to the minimal amount of circular polarization, the ellipses appear as line segments. Fig. 1C) shows the angle of the tilt of the polarization in terms of the Stokes parameters $Q$ and $U$, with the same resolution as the intensity image.

What we would like to draw attention to in Fig. 1 is the axial pattern over the celestial hemisphere of the polarization ellipses. This pattern agrees quite well, at least in the central half of the sky, with the polarization aberration pattern determined in Carozzi \& Woan (2009a) for the case of crossed short dipole elements. A similar effect was observed in Wijnholds (2005), albeit in the LOFAR ITS array at much lower frequencies. The explanation for the aberration is that radiation from the largely unpolarized sky is projected onto the dipole moments of the LBA, which are parallel to the ground. Although this aberration can be partially removed, as shown in Carozzi \& Woan (2009b), the instrumental polarization exhibited here (i.e. the ratio of the polarized power, as indicated in Fig. 1 by the size of the polarization ellipse, to the total power) is directly related to IXR. Therefore full calibration is only possible if the polarization ellipses are sufficiently small. Clearly, in Fig. 1, the IXR is rotationally symmetric and best at zenith, so high dynamic range imaging will only be possible around zenith.

Fig. 2 shows the correlation, or normalised inner-product (measure of orthogonality), of the two polarized components of a tapered slot array tile over the celestial hemisphere plotted in horizontal (Elevation-Altitude) coordinates. The data comes from a simulation described in Maaskant et al. (2010) of an EMBRACE-like tile called VALARRAY. EMBRACE is one of the AA technologies considered for the SKA.

What we would like to draw attention to in Fig. 2 is the pattern with a four-fold symmetry, where the orthogonality is best along the principle planes of the array elements, that is the E-planes and H-planes of the vivaldi feed elements. The 


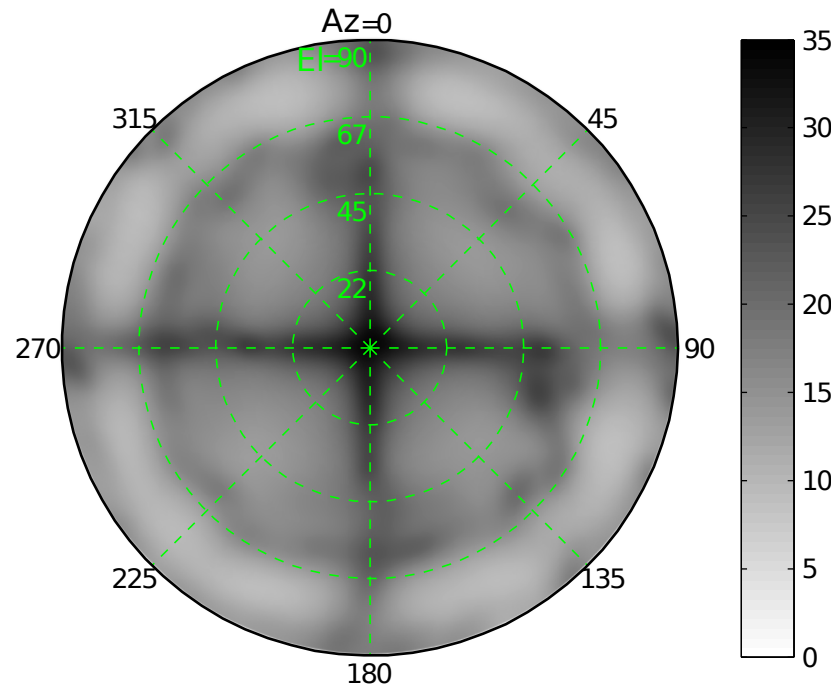

Fig. 2: Orthogonality of polarization components in a dual-polarized tapered slot array tile.

non-orthogonality (degeneracy) along the diagonal D-planes is typically for this type of AA (Schaubert \& Chio 2000).

\section{Polarization diversity}

As we have seen in the previous sections, polarimetric aberrations are the rule rather than the exception over wide fields, and we discussed that these aberrations can only be corrected up to the IXR corresponding to the aberrations. We will now consider what can be done to improve the SKA polarimetry over wide fields.

Up to now we have made two assumptions on the current design of the SKA:

1. that there are only two polarized far-field radiation vectors (corresponding to the two elements of the dual-polarized antennas) for all the AAs,

2. that the AAs are fixed mount (i.e. the pointing is done by electronic "polarimetric" beamforming)

3. the boresight of the AAs is towards zenith.

Assumptions 1 and 2 imply that, instantaneously, the SKA will only have two polarization vectors for polarimetry of an arbitrary source, but as we have seen these polarization vectors can be degenerate. This is regardless of how many dual-polarized antennas we have in the AAs; so even if the SKA has millions of elements, as long as they are all the same type of dualpolarized feed and the same orientation, there will only be two polarization vectors for polarimetry. On the other hand, if there were to be more than two polarization vectors per image pixel, then there would be less of a chance that all the polarization vectors would be degenerate. This suggests that the design of the SKA may benefit from having more than just the homogeneous array of dual-polarized elements per frequency range.

In particular, one should strive for polarization diversity. By this we mean having a wider range of orientations of the AAs. There are many ways in which polarization diversity can be implemented. Unfortunately, due to the scale of the SKA, many polarization diversity schemes are prohibitively expensive, and therefore we will just mention one such scheme that is simple and almost without cost. It is simply to rotate some of the AAs with respect to each other, and in particular to rotate half of the AA stations by $45^{\circ}$ with respect to the other half. Polarization diverse AA can be viewed as a type of heterogeneous array as defined in Hamaker (2000).

Our reason for mentioning this simple scheme is not that it is optimal, but it illustrates a cost-effective way of mitigating the risks involved in wide-field polarimetry.

\subsection{Polarimetric beamforming with dual-polarized elements}

We will now look at our proposed scheme for simple polarization diversity in more detail, but before we can do that we will have to formulate the theory for polarimetric beamforming, i.e., beamforming with polarized radiation. To simplify, we start with the default scenario: dual-polarized elements.

Basically, polarimetric beamforming with dual-polarized elements is a simple generalization of the usual array factor theorem in antenna theory to include an additional polarized element. We call the two polarized elements of the dual-polarized antennas $X$ and $Y$ and their respective far-field radiation vector patterns $\mathbf{F}^{(X)}(\mathbf{k})$ and $\mathbf{F}^{(Y)}(\mathbf{k})$. Disregarding the obstruction of the array structure on the propagation, two far-field radiation vector patterns $\mathbf{F}_{\text {tot }}^{(X)}$ and $\mathbf{F}_{\text {tot }}^{(Y)}$ (associated here with the $X$ and $Y$ as channels) are formed according to

$$
\begin{aligned}
& \mathbf{F}_{\text {tot }}^{(X)}(\mathbf{k})=a^{(X X)}(\mathbf{k}) \mathbf{F}^{(X)}(\mathbf{k})+a^{(X Y)}(\mathbf{k}) \mathbf{F}^{(Y)}(\mathbf{k}) \\
& \mathbf{F}_{\text {tot }}^{(Y)}(\mathbf{k})=a^{(Y Y)}(\mathbf{k}) \mathbf{F}^{(Y)}(\mathbf{k})+a^{(Y X)}(\mathbf{k}) \mathbf{F}^{(X)}(\mathbf{k})
\end{aligned}
$$

where

$$
\begin{aligned}
& a^{(X X)}(\mathbf{k})=\sum_{i=1}^{N} w_{i}^{(X X)} \exp \left(\mathrm{ik} \cdot \mathbf{r}_{i}^{(X)}\right) \\
& a^{(X Y)}(\mathbf{k})=\sum_{i=1}^{N} w_{i}^{(X Y)} \exp \left(\mathrm{i} \mathbf{k} \cdot \mathbf{r}_{i}^{(Y)}\right) \\
& a^{(Y X)}(\mathbf{k})=\sum_{i=1}^{N} w_{i}^{(Y X)} \exp \left(\mathrm{ik} \cdot \mathbf{r}_{i}^{(Y)}\right) \\
& a^{(Y Y)}(\mathbf{k})=\sum_{i=1}^{N} w_{i}^{(Y Y)} \exp \left(\mathrm{i} \mathbf{k} \cdot \mathbf{r}_{i}^{(X)}\right)
\end{aligned}
$$

in which $w_{i}^{(.)}$are the complex weights on the $N$ individual elements per polarization and $\mathbf{r}_{i}^{(.)}$are the antenna positions. Most of these quantities are functions of the wave vector, $\mathbf{k}=\omega / c\left(s_{x}, s_{y}, s_{z}\right)$, which represents the frequency, $\omega$, and the look-direction, $\left(s_{x}, s_{y}, s_{z}\right)$, of the radiation. If there was no mutual coupling between polarized elements $X$ and $Y$, then $w_{i}^{(X Y)}$ and $w_{i}^{(Y X)}$ could be set to zero, and then $\mathbf{F}_{\text {tot }}^{(X)}$ and $\mathbf{F}_{\text {tot }}^{(Y)}$ would just be functions of $\mathbf{F}^{(X)}$ and $\mathbf{F}^{(Y)}$ respectively.

From Eq.s (1) one can draw the important conclusion that for an array of identical dual-polarized antennas, there are only two vector-valued degrees of freedom, namely $\mathbf{F}^{(X)}$ and $\mathbf{F}^{(Y)}$, 
per look-direction. So if these are parallel to each other, then the two are degenerate for purposes of polarimetric beamforming. In terms of SKA polarimetry, this mean regardless of how large $N$ is, there are only two polarimeter vectors per image pixel and these may well be degenerate.

As a simple example of polarimeter vectors $\mathbf{F}^{(X)}$ and $\mathbf{F}^{(Y)}$, consider a pair of crossed short (electric) dipoles. The effective antenna length of the short dipole can be modeled as a constant vector (i.e. independent of look-direction) along the linear extent of the dipole (Carozzi \& Woan 2009a). If one places a short dipole along $x$ and $y$, both orthogonal to zenith, then removes the radial component of their effective antenna length vectors, one obtains the crossed dipole's polarimeter vectors $\mathbf{F}^{(X)}(\mathbf{k})$ and $\mathbf{F}^{(Y)}(\mathbf{k})$ (which now depend on look-direction). These polarimeter vectors are shown in Fig. 3 over a hemisphere with zenith at the centre of the field. The $X$ channel is red and the $Y$ channel is blue, and they are plotted as vectors in the tangent plane of celestial sphere. We have used a azimuthal equidistant projection (i.e. we are using the polar coordinates elevation-altitude) so that the angle between the two polarimeter vectors is maintained in the plot. Thus we can see that at zenith, the crossed short dipoles appear orthogonal, but towards the horizon the polarimeter vectors are progressively non-orthogonal, and at the horizon they are degenerate. This simple example

The vector plot is layed over a grey-scale plot of the polarimeter's IXR, which shares the same coordinate system as the polarimeter vectors, but which is plotted at a higher resolution. The IXR confirms quantitatively what we quantitatively concluded with regards to the degeneracy of the polarimeter vectors. The IXR plot clearly shows that this effect is fully rotationally symmetric. This is somewhat surprising, although it was already pointed out in Carozzi \& Woan (2009b), considering that the E-plane and H-plane of a short dipole are very different from each other (one is omnidirectional, the other is a cosine respectively). One should note that the IXR is not simply a measure of orthogonality, but includes the detrimental effect of differential channel gains.

\subsection{Polarimetric beamforming with polarization diversity}

Now consider we have at least two identical dual-polarized antenna elements. Next, rotate one of the two by $45^{\circ}$ with respect to the other. If both these dual-polarized elements are used for polarimetry, then this simple array of two distinct dualpolarized antenna elements would constitute a simple example of a polarization-diverse radio interferometer.

The situation is sketched in Fig. 4. The dual-polarized element of the left is taken as the reference. For sake of simplicity we show it as crossed dipoles aligned with the cardinal directions: East-West and North-South are the nominal directions for the $X$ (red) and $Y$ (blue) channels respectively. We take the far-field radiation vector patterns associated with these two antennas to be $\mathbf{F}^{(X)}(\mathbf{k})$ and $\mathbf{F}^{(Y)}(\mathbf{k})$. The polarization diverse element is shown on the right in Fig. 4 in solid magenta and green

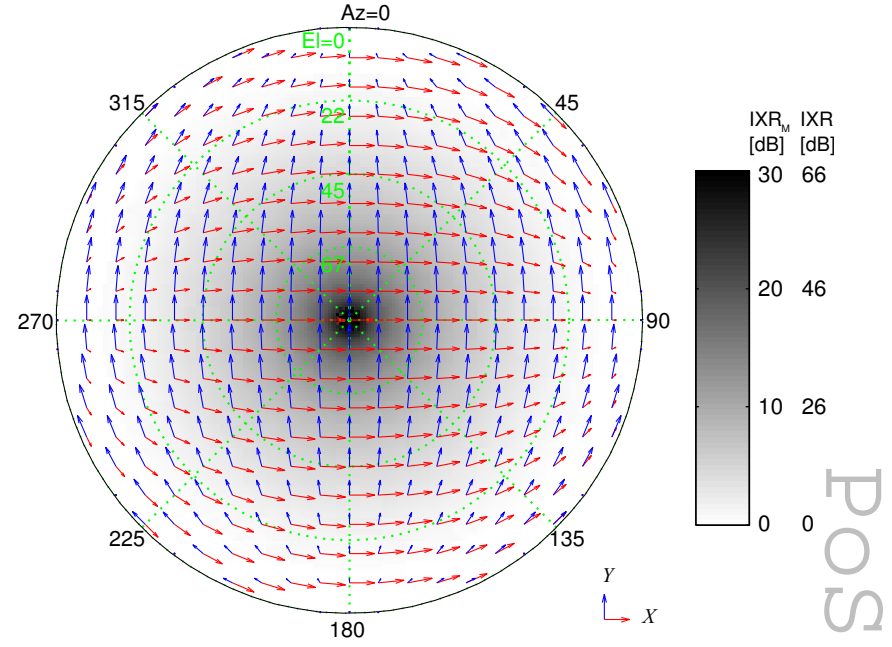

Fig. 3: The polarimeter vectors of crossed short dipoles (no beamforming) and the corresponding polarimeter IXR over a hemisphere. It can be seen as a model of the LOFAR LBA, which despite its simplicity does predict rotational symmetry of the instrumental polarization which is indeed found in Fig. 1.
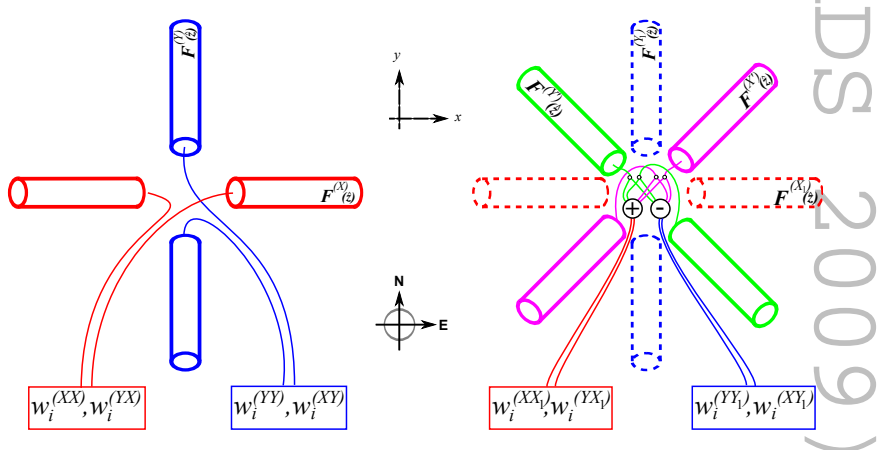

Fig. 4: Sketch of dual-polarized array elements (solid lines) with simple polarization diversity: the dual-polarized element on the right is a rotated version of the dual-polarized element on the left. The adder and subtracter in the sketch can be implemented either in back-end electronics or in the beamforming software.

lines, and the corresponding radiation vector patterns are denoted $\mathbf{F}^{\left(X^{\prime}\right)}(\mathbf{k})$ and $\mathbf{F}^{\left(Y^{\prime}\right)}(\mathbf{k})$.

The relationship between these two sets of vector fields is

$$
\begin{aligned}
& \mathbf{F}^{\left(X^{\prime}\right)}=\mathbf{R}_{\times} \mathbf{F}^{(X)}\left(\mathbf{R}_{\times}^{-1} \mathbf{k}\right) \\
& \mathbf{F}^{\left(Y^{\prime}\right)}=\mathbf{R}_{\times} \mathbf{F}^{(Y)}\left(\mathbf{R}_{\times}^{-1} \mathbf{k}\right)
\end{aligned}
$$

where

$$
\mathbf{R}_{\times}=\frac{1}{\sqrt{2}}\left(\begin{array}{cc}
1 & -1 \\
1 & 1
\end{array}\right)
$$

is the $45^{\circ}$ rotation matrix.

In principle this is a sufficient scheme to achieve an array with polarization diversity. However, it does have a minor complication in that the $X$ and $Y$ channels of the rotated dualpolarized antenna are no longer nominally along the $x$ - and $y$-directions. For the purposes of signal processing and polarimetric beamforming, this can be simplified by including some 
simple electronics on the back-end of the dual-polarized antenna element, so that the output channels are rotated electronically back to the reference coordinate system. This is can be implemented using an adder and a differencer as shown in Fig. 4. The effect of this electronic rotation of the output channels amounts to introducing two new polarimeter vectors

$$
\begin{aligned}
& \mathbf{F}^{\left(X_{1}\right)}=\left(\mathbf{F}^{\left(X^{\prime}\right)}-\mathbf{F}^{\left(Y^{\prime}\right)}\right) / \sqrt{2} \\
& \mathbf{F}^{\left(Y_{1}\right)}=\left(\mathbf{F}^{\left(X^{\prime}\right)}+\mathbf{F}^{\left(Y^{\prime}\right)}\right) / \sqrt{2} .
\end{aligned}
$$

These two virtual antennas are shown in Fig. 4 with dashed red and blue lines, implying that the virtual antennas look to the system as though they were similar to the $X$ and $Y$ channels.

The end result of this is a simplification in the operation of this polarizationally diverse array. The polarimetric beamforming equations are now

$$
\begin{aligned}
\mathbf{F}_{\text {tot }}^{(X)}= & a^{(X X)} \mathbf{F}^{(X)}+a^{(X Y)} \mathbf{F}^{(Y)} \\
& +a^{\left(X X_{1}\right)} \mathbf{F}^{\left(X_{1}\right)}+a^{\left(X Y_{1}\right)} \mathbf{F}^{\left(Y_{1}\right)} \\
\mathbf{F}_{\text {tot }}^{(Y)}= & a^{(Y X)} \mathbf{F}^{(X)}+a^{(Y Y)} \mathbf{F}^{(Y)} \\
& +a^{\left(Y X_{1}\right)} \mathbf{F}^{\left(X_{1}\right)}+a^{\left(Y Y_{1}\right)} \mathbf{F}^{\left(Y_{1}\right)}
\end{aligned}
$$

where

$$
\begin{aligned}
& a^{\left(X X_{1}\right)}(\mathbf{k})=\sum_{i=N+1}^{2 N} w_{i}^{\left(X X_{1}\right)} \exp \left(\mathrm{ik} \cdot \mathbf{r}_{i}^{\left(X_{1}\right)}\right) \\
& a^{\left(X Y_{1}\right)}(\mathbf{k})=\sum_{i=N+1}^{2 N} w_{i}^{\left(X Y_{1}\right)} \exp \left(\mathrm{ik} \cdot \mathbf{r}_{i}^{\left(Y_{1}\right)}\right) \\
& a^{\left(Y X_{1}\right)}(\mathbf{k})=\sum_{i=N+1}^{2 N} w_{i}^{\left(Y X_{1}\right)} \exp \left(\mathrm{ik} \cdot \mathbf{r}_{i}^{\left(X_{1}\right)}\right) \\
& a^{\left(Y Y_{1}\right)}(\mathbf{k})=\sum_{i=N+1}^{2 N} w_{i}^{\left(Y Y_{1}\right)} \exp \left(\mathrm{ik} \cdot \mathbf{r}_{i}^{\left(Y_{1}\right)}\right)
\end{aligned}
$$

and $w_{i}^{(.)}$for $i=N+1, \ldots, 2 N$ are the new sets of weights for the rotated (forward physically and backward electronically) set of dual-polarized elements.

As an example of this, we take as the basic element a Vivaldi-type feed. Using a simple analytic model for the polarimeter vectors, we make the same sort of plot as in Fig. 3, one for each of the two orientation of the dual-polarized elements. The results are shown in Fig. 5. The upper plot shows the polarimeter in the reference orientation. Compared with Fig. 3, one sees that the pattern is only four-fold rotationally symmetric. The IXR is best along the E-plane and the H-plane of the crossed vivaldi feeds, but worst in the D-planes (45 degree diagonal planes).

The pattern for an identical, but rotated, dual-polarized vivaldi feed is in the lower part of Fig. 5. The IXR plot is identical to corresponding plot in the upper part of the figure, as we expected. The polarimeter vector plots in the two cases are the same on axis (zenith), but off-axis the become gradually more different as one goes towards the horizon. This is the intended effect, and demonstrates that the simple scheme for polarization diversity proposed here does indeed provide a diverse set

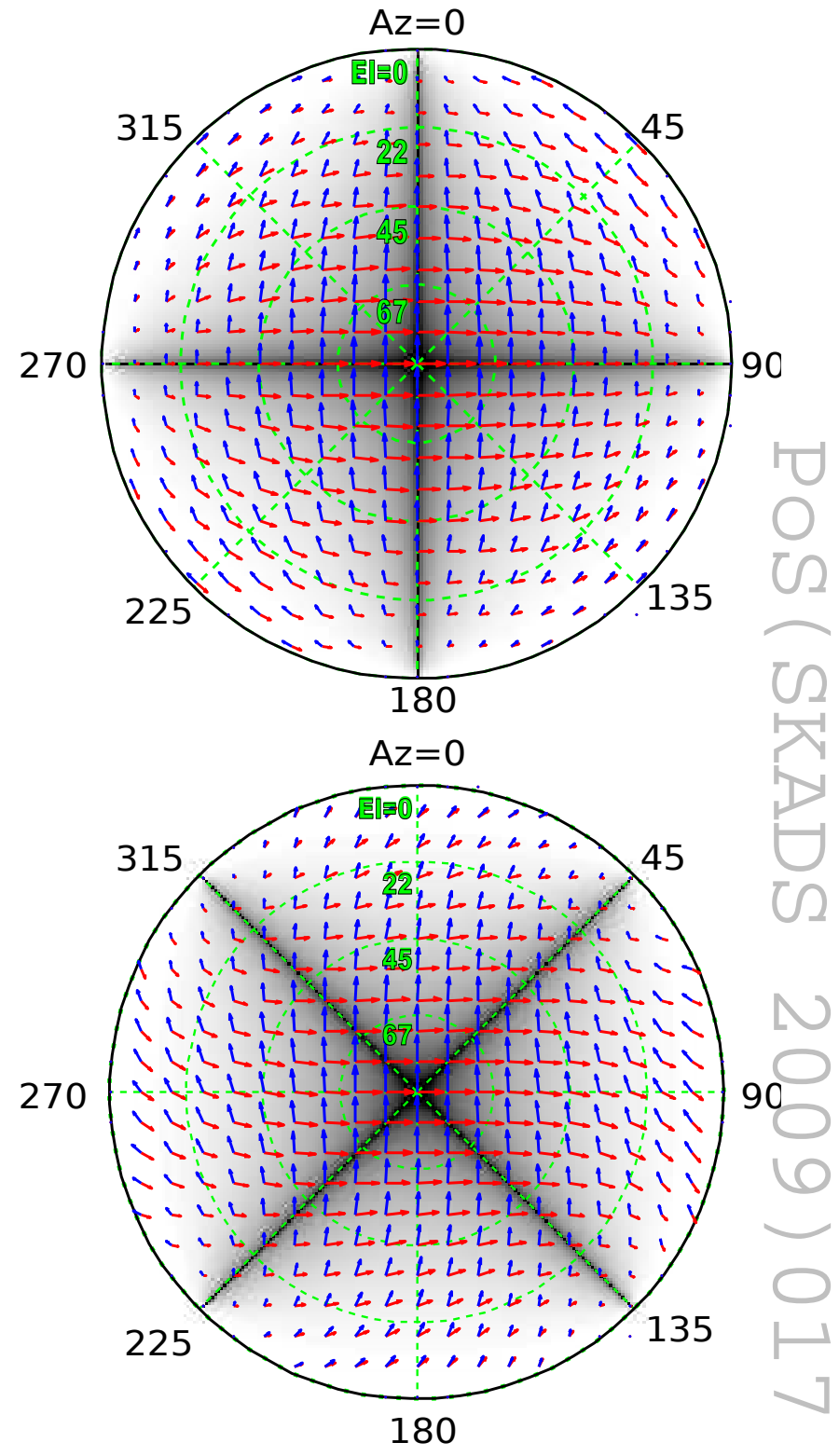

Fig. 5: Same type plots and scales as in Fig. 3, but for Vivalditype feeds with two different alignments: along cardinal directions North-South and East-West (uppermost), and rotated 45 degrees (lowermost).

of polarimeter vectors to cover the field of view thereby avoiding regions where polarimetry may be poor. Of course, if the patterns were rotationally symmetric such as in the case of the LOFAR LBA, Fig. 1, or crossed short dipoles, Fig. 3, then this type of polarization diversity would not have that much effect.

Note that a dual-polarized antenna rotated around boresight does not exhibit any loss in the total intensity since there is never any polarization mismatch, thus no power can be lost polarimetrically. Thus polarization diversity also has the added benefit of increasing the overall received source power in the telescope. 


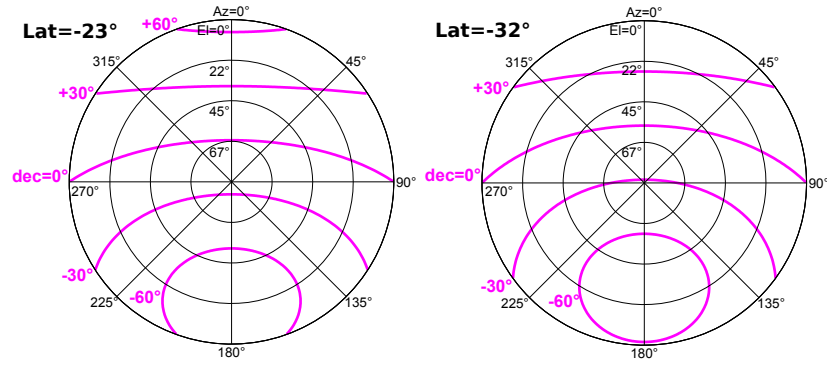

Fig. 6: Loci of constant declination plotted in a polar plot as a function of polar coordinates.

\section{Earth's rotation and polarization diversity}

Our discussion of polarization diversity has mainly focused on the instantaneous polarimetry, that is, the snap-shot mode of the SKA. Obviously, the assumption that the AA does not move means the diurnal rotation of the Earth will itself rotate the element. Thus the SKA will have some polarization diversity for free. However, this does not make the simple polarization diversity scheme discussed in the previous section superfluous. First the diurnal rotation does not apply for snap-shot imaging, which is important for transients. Furthermore the diurnal rotation is with respective to the poles of the celestial sphere, while the boresight of the AAs of the SKA will be towards zenith, so the two types polarization diversity through rotation will be somewhat complementary.

The effect of the diurnal rotation on the polarization diversity of a dual-polarized element can be grasped by considering the trajectory of the fixed sources on celestial sphere in the same sort of polar plots we used previously for the IXR (and orthogonality) of various polarimeters. These are shown in Fig. 4 for two different latitudes, which correspond roughly to the two candidate sites for the SKA. The different magenta lines are for different source declinations. If one overlays these plots over the plots in Fig.s 2, 3, and 5, one sees how the sources at different declinations move through the different beam patterns. One finds that for Vivaldi-type polarimeter patterns, the best alignment is along the cardinal directions, since then one is guaranteed that all visible sources will pass the E- and H-planes of the feeds at the meridian transit. In any case, it is clear that the poles are still sufficiently far away from the boresight, where the polarimetry is best, that the SKA stands to benefit from the polarization diversity scheme suggested here.

\section{Conclusion}

We find that wide-field polarimetry with the AA technology currently considered for the SKA, stands to benefit from polarization diversity. A simple cost-effective scheme for polarization diversity in SKA was proposed and found to improve not only polarimetry but even the overall power received from sources.

Acknowledgements. We wish to thank Stefan Wijnholds for the LOFAR CS10 data and comments on this paper.

\section{References}

W. A. van Cappellen, et al. Low Band Antenna: Architectural Design Document. Technical Report ADD-009, ASTRON, 21 March 2007. 395(3):1558-1568, 2009.

T. D. Carozzi and G. Woan. A generalized measurement equation and van Cittert-Zernike theorem for wide-field radio astronomical interferometry. Monthly Notices of the Royal Astronomical Society,

T. D. Carozzi and G. Woan. A fundamental figure of merit for radio polarimeters. submitted to IEEE trans. Ant. Prop. special issue, 2009.

J. Hamaker. Self-Calibration in the Ska: Dealing with Inherently Strong Instrumental Polarization. In A. B. Smolders \& M. P. van Haarlem, editor, Perspectives on Radio Astronomy: Technologies for Large Antenna Arrays, pages 323-+, 2000.

R. Maaskant, et al. Analysis of Large Microstrip-Fed Tapered Slot Antenna Arrays by Combining Electrodynamic and Quasi-Static Field Models. submitted to IEEE trans. Ant. Prop. special issue.

D. H. Schaubert and T. H. Chio. Wideband Vivaldi Arrays for Large Aperture Antennas. In A. B. Smolders \& M. P. van Haarlem, editor, Perspectives on Radio Astronomy: Technologies for Large Antenna Arrays, pages 49-+, 2000.

R. T. Schilizzi, et al. Preliminary Specifications for the Square Kilometre Array. Technical report SKA memo 100, SKA Program Development Office, 10 December 2007.

S. J. Wijnholds. ITS results 2005: Filling in the gaps. Technical Report RPT-056, ASTRON, 23 September 2005. 